\title{
Anisotropic Absorption of Pure Spin Currents
}

\author{
A. A. Baker, ${ }^{1,2}$ A. I. Figueroa, ${ }^{1}$ C. J. Love, ${ }^{1,3}$ S. A. Cavill, ${ }^{3,4}$ T. Hesjedal, ${ }^{2,4, *}$ and G. van der Laan ${ }^{1, \dagger}$ \\ ${ }^{1}$ Magnetic Spectroscopy Group, Diamond Light Source, Didcot OX11 ODE, United Kingdom \\ ${ }^{2}$ Department of Physics, Clarendon Laboratory, University of Oxford, Oxford OX1 3PU, United Kingdom \\ ${ }^{3}$ Department of Physics, University of York, York YO10 5DD, United Kingdom \\ ${ }^{4}$ Diamond Light Source, Didcot OX11 ODE, United Kingdom
}

(Received 1 October 2015; published 27 January 2016)

\begin{abstract}
Spin transfer in magnetic multilayers offers the possibility of ultrafast, low-power device operation. We report a study of spin pumping in spin valves, demonstrating that a strong anisotropy of spin pumping from the source layer can be induced by an angular dependence of the total Gilbert damping parameter, $\alpha$, in the spin sink layer. Using lab- and synchrotron-based ferromagnetic resonance, we show that an in-plane variation of damping in a crystalline $\mathrm{Co}_{50} \mathrm{Fe}_{50}$ layer leads to an anisotropic $\alpha$ in a polycrystalline $\mathrm{Ni}_{81} \mathrm{Fe}_{19}$ layer. This anisotropy is suppressed above the spin diffusion length in $\mathrm{Cr}$, which is found to be $8 \mathrm{~nm}$, and is independent of static exchange coupling in the spin valve. These results offer a valuable insight into the transmission and absorption of spin currents, and a mechanism by which enhanced spin torques and angular control may be realized for next-generation spintronic devices.
\end{abstract}

DOI: 10.1103/PhysRevLett.116.047201

The generation and detection of spin currents is at the foundation of spintronics, being integral to many proposals for new memory and logic devices [1-3]. The pure spin current emitted by a ferromagnet (FM) undergoing ferromagnetic resonance (FMR) [4] is one candidate, efficiently producing torques without necessitating a charge current. The spin current can persist across a normal metal (NM), either returning to the source FM or flowing through to a second FM, where it is absorbed and induces precession through the spin transfer torque (STT) [5]. Spin pumping can be observed by measuring increased damping (through increased FMR linewidth) due to an outflow of angular momentum from the source FM [6,7], by the inverse spin Hall effect in the sink layer [8], or by layer-specific measurements of precession or spin accumulation using x-ray magnetic circular dichroism (XMCD) [9-11].

The strength of the dynamic interaction that arises due to the pumped spin current is influenced by the transmission of spins across the FM-NM interfaces (the spin mixing conductance), and by the diffusion length of the spin current in the NM spacer layer [12,13]. The diffusion length ranges from under a nanometer in an insulator such as $\mathrm{MgO}$ [8] or $\mathrm{SrTiO}_{3}$ [14] to $>100 \mathrm{~nm}$ in good conductors such as $\mathrm{Ag}$ [15]. The angular dependence of the resonance linewidth contains several contributions, including two-magnon scattering [16], mosaic broadening $[17,18]$, and anisotropic Gilbert damping arising due to field dragging [18].

Published by the American Physical Society under the terms of the Creative Commons Attribution 3.0 License. Further distribution of this work must maintain attribution to the author(s) and the published article's title, journal citation, and DOI.
Spin pumping can have a pronounced angular dependence, arising from the relative alignment of the two magnetic layers and the magnitude of precession [19-21]. In this case, antiparallel alignment leads to more efficient absorption of the spin current as compensation of the pumped angular momentum drops [22]. Similarly, damping is higher when the precession of the magnetizations is out of phase. In trilayers with static coupling, damping can be further enhanced due to a change in the optical and acoustic character of the resonances of the two layers [23]. Furthermore, if the FM layers are simultaneously at resonance, the additional damping due to the pumped spin currents can mutually cancel [24]. Stray fields from domain walls in the spin sink layer may also increase damping through an effective dipolar field [25].

In this Letter we present a study of a new mechanism for angular control of the spin current: anisotropic damping in the spin sink layer that can affect spin pumping from the source layer. Using $\mathrm{Co}_{50} \mathrm{Fe}_{50} / \mathrm{Cr} / \mathrm{Ni}_{81} \mathrm{Fe}_{19}$ spin valves with variable $\mathrm{Cr}$ thicknesses, static exchange can be tuned to prefer ferromagnetic or antiferromagnetic alignment. Analysis of the resonance linewidth indicates anisotropic spin pumping in the NiFe layer, correlated with the general damping anisotropy in CoFe. This demonstrates that transmission of the spin current across the NM-FM interface into the spin sink layer is affected by damping within the FM layer. Layer resolved measurements show that the spin current pumped from the NiFe layer exerts an anisotropic torque on the $\mathrm{CoFe}$ layer magnetization, highlighting the potential for a new mechanism to realize angular control of spin currents.

Spin valve samples were prepared by molecular beam epitaxy (MBE) in a mini-MBE system [26] on epiready $\mathrm{MgO}$ (001) substrates. The full structure is 
$\mathrm{MgO} / \mathrm{Co}_{50} \mathrm{Fe}_{50}(5) / \mathrm{Cr}\left(t_{\mathrm{Cr}}\right) / \mathrm{Ni}_{81} \mathrm{Fe}_{19}(5) / \mathrm{Ag}(2)$ (thickness is in nanometers), with $t_{\mathrm{Cr}}=1,1.5,2,5,10 \mathrm{~nm}$. The sample was held at $500{ }^{\circ} \mathrm{C}$ for deposition of stoichiometric $\mathrm{Co}_{50} \mathrm{Fe}_{50}$; epitaxial growth was observed using reflection high energy electron diffraction. The sample was then cooled to room temperature for the deposition of the $\mathrm{Cr}$ and $\mathrm{Ni}_{81} \mathrm{Fe}_{19}$ layers to inhibit intermixing of the layers and to ensure that the $\mathrm{Ni}_{81} \mathrm{Fe}_{19}$ layer has an isotropic, polycrystalline structure.

Magnetometry measurements were carried out using a SQUID-vibrating sample magnetometer (VSM). FMR measurements were performed using a vector network analyzer and octupole electromagnet. Real and imaginary components of the microwave transmission parameter, $S_{12}$, were measured as a function of the magnetic field (strength and angle) and the frequency. Plotting the resonant field as a function of the frequency and the bias field angle yielded anisotropy and exchange coupling parameters, with the total Gilbert damping, $\alpha$, extracted from the frequency dependence of the linewidth using Eq. (3).

XMCD measurements were performed on beam line I10 at the Diamond Light Source (UK) and beam line 4.0.2 at the Advanced Light Source (U.S.). The magnetic sample is excited by microwaves phase locked to the x-ray repetition rate, resulting in a steady precession about the effective field close to the FMR conditions. The oscillating magnetization component along the $\mathrm{x}$-ray direction is probed using XMCD. Element specificity is obtained by tuning the $\mathrm{x}$-ray energy to the absorption edge of the element of interest. For full details of the X-ray detected FMR (XFMR) methodology, we refer the reader to Ref. [27]. All XFMR measurements were performed at the $\mathrm{Ni}$ and $\mathrm{Co} L_{3}$ edges. Element-specific hysteresis measurements were performed at the $\mathrm{Co}, \mathrm{Ni}$, and $\mathrm{Fe} L_{3}$ edges by sweeping the magnetic field from +100 to $-100 \mathrm{mT}$ and back at both $\mathrm{x}$-ray helicities.

Figure 1 shows hysteresis loops measured by SQUIDVSM (left column) and element-specific XMCD hysteresis (right column) for samples with $t_{\mathrm{Cr}}=1,1.5$, and $2 \mathrm{~nm}$, in which three distinct coupling regimes can be clearly observed. For the $1 \mathrm{~nm} \mathrm{Cr}$ layer [see Fig. 1(a)] the strong FM interaction between the layers aligns the two magnetizations, leading to a single switching step with a coercive field of $2 \mathrm{mT}$. For $t_{\mathrm{Cr}}=1.5 \mathrm{~nm}$ [see Fig. 1(c)], however, the coupling becomes antiferromagnetic (AFM), with the $\mathrm{NiFe}$ and $\mathrm{CoFe}$ layers preferring antiparallel alignment. For $t_{\mathrm{Cr}}=2 \mathrm{~nm}$ [see Fig. 1(e)] there is no coupling and two distinct switching steps as the layers reverse independently. Precise tuning of the static exchange strength, $A_{\mathrm{ex}}$, allows the contributions of static and dynamic exchange to anisotropic damping to be separated.

Figures 2(a) and 2(b) show FMR measurements of fieldfrequency transmission maps along the easy and hard axis of the $\mathrm{CoFe}$, respectively. The anisotropic resonance showing the inflection point on the hard axis is dominated
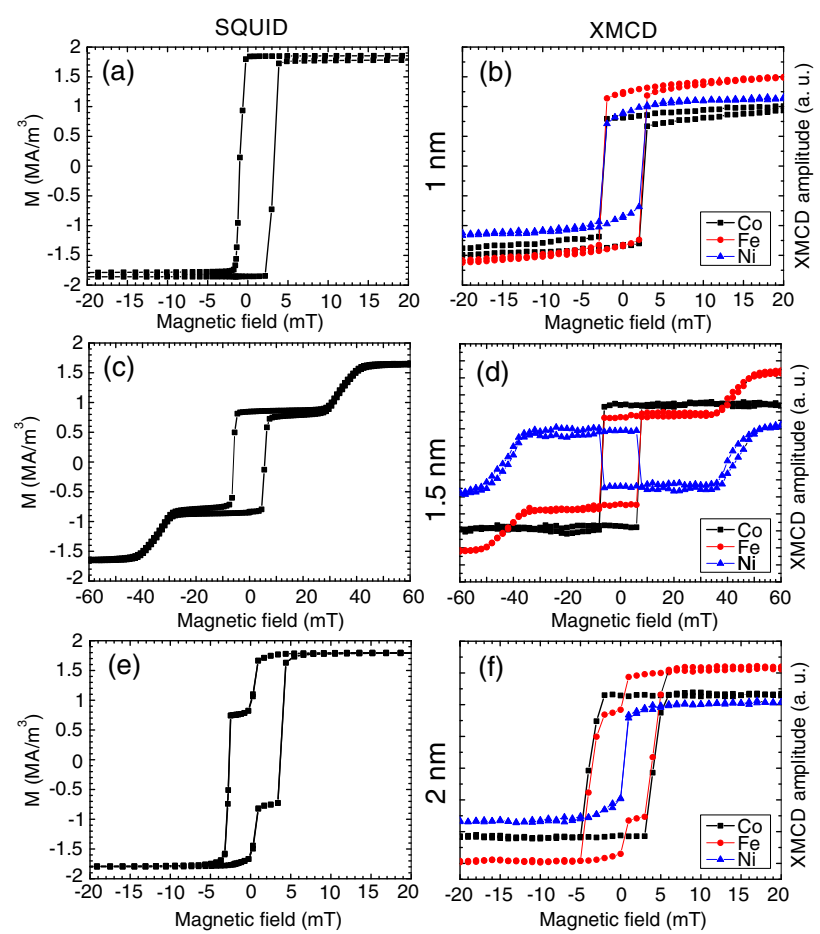

FIG. 1. SQUID-VSM and XMCD hysteresis measurements of spin valves with $t_{\mathrm{Cr}}=1 \mathrm{~nm}(\mathrm{a}),(\mathrm{b}), 1.5 \mathrm{~nm}$ (c),(d), and $2 \mathrm{~nm}(\mathrm{e})$, (f). As the thickness of the Cr layer increases from 1 to $1.5 \mathrm{~nm}$ the coupling changes from FM to AFM before vanishing at $t_{\mathrm{Cr}}=2 \mathrm{~nm}$. XMCD reveals the element-specific steps in the switching, particularly the AFM coupling of the Ni at low fields for $t_{\mathrm{Cr}}=1.5 \mathrm{~nm}$.

by the $\mathrm{CoFe}$, whereas the more isotropic mode stems from the $\mathrm{NiFe}$ layer. The resonance frequency, $\omega=2 \pi f$, for in-plane magnetization is determined by the Kittel equation [28]

$$
\omega=\gamma \sqrt{H_{\text {eff }}\left(H_{\text {eff }}+M\right)},
$$

with $\gamma$ being the gyromagnetic ratio, $M$ the magnetization, and $H_{\text {eff }}$ the effective field, composed of Zeeman, anisotropy, and exchange coupling fields. The exchange coupling field is defined as $[29,30]$

$$
\mu_{0} H_{\mathrm{ex}}^{i}=\frac{A_{\mathrm{ex}}}{M_{S}^{i} d^{i}} \cos \left(\phi_{M}^{i}-\phi_{M}^{j}\right),
$$

with $A_{\text {ex }}$ being the interlayer exchange coupling parameter (which is the same for both layers), $d^{i}$ the thickness, and $\phi_{M}^{i}$ the alignment of the magnetization in layer $i$. The value of the interlayer exchange was determined by fitting the resonant field for a range of angles and fields, using $M_{s}$ determined by SQUID VSM. Example results are shown in Fig. 2(c). The CoFe resonance field varies by $\pm 50 \mathrm{mT}$ due to its strong magnetocrystalline anisotropy, while the NiFe resonance field has a much weaker variation of $\pm 5 \mathrm{mT}$, driven by a small magnetocrystalline anisotropy and exchange coupling with the CoFe layer [31]. Figure 2(d) 

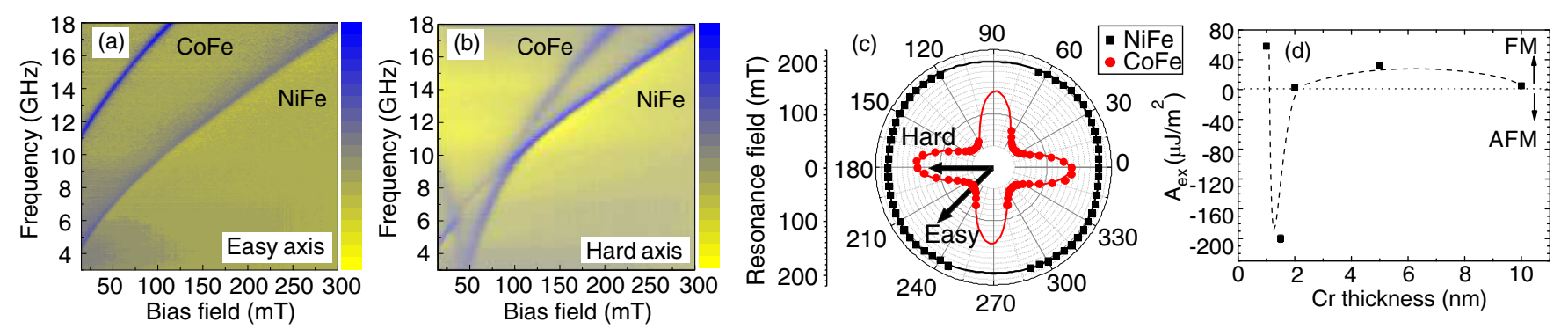

FIG. 2. (Left panels) Field vs frequency-transmission maps, showing Kittel curves of two resonances of the $t_{\mathrm{Cr}}=1 \mathrm{~nm}$ sample with the bias field aligned along the easy (a) and hard (b) axis of the CoFe. (c) Angular dependence of the two resonances at driving frequency $14 \mathrm{GHz}$. Solid lines are fits to the data using Eq. (1). (d) Extracted interlayer exchange coupling, $A_{\text {ex }}$, as a function of Cr interlayer thickness, showing FM and AFM coupling before being suppressed for a large $t_{\mathrm{Cr}}$. Error bars are comparable to the point size.

shows $A_{\mathrm{ex}}$ as a function of $t_{\mathrm{Cr}}$, and the expected oscillatory behavior between FM and AFM coupling is observed [32].

The resonance linewidth, $\Delta H$, has both intrinsic (Gilbert) and extrinsic (inhomogeneous broadening) contributions and is given by [33]

$$
\Delta H=\Delta H_{0}+\frac{\alpha 4 \pi f}{\gamma},
$$

with $\Delta H_{0}$ being the inhomogeneous broadening and the total Gilbert damping $\alpha=\alpha_{0}+\alpha_{\mathrm{sp}}$. Further information on fitting can be found in Ref. [31]. The spin pumping damping $\alpha_{\mathrm{sp}}$ can be inferred by plotting $\alpha$ as a function of interlayer thickness, as the damping associated with a bare FM, $\alpha_{0}$, is not affected by interlayer thickness. This is shown in Figs. 3(a) and 3(b) for the NiFe and CoFe layers, respectively. An exponential decrease in damping with increasing $\mathrm{Cr}$ thickness is observed, indicative of spin pumping. This arises as spins pumped from the precessing ferromagnetic layer are unable to reach the second FM, instead scattering in the spacer layer or flowing back to the spin source, where they exert an antidamping torque. Fits to the data were performed using the procedure outlined by Kardasz and Heinrich [15], giving a spin diffusion length of $8 \mathrm{~nm}$ in Cr. This is somewhat less than the $13 \mathrm{~nm}$ reported by Du et al. [34] using inverse spin Hall effect measurements.

Strikingly, Fig. 3(a) shows that there is a significant anisotropy of damping in the polycrystalline $\mathrm{NiFe}$ layer, for $t_{\mathrm{Cr}} \leq 2 \mathrm{~nm}$. The suppression of the anisotropy for higher values of $t_{\mathrm{Cr}}$ suggests that it arises from an interaction with the CoFe layer. Figure 3(b) shows that damping is also anisotropic in the $\mathrm{CoFe}$ layer, being significantly higher when the bias field is aligned along the magnetocrystalline hard axis. This could be indicative of inhomogeneous damping or two-magnon scattering arising from the coordination of defects in the crystal structure $[18,35]$. The $\mathrm{NiFe}$ layer is examined in Fig. 3(c) in more detail, showing the angular dependence of Gilbert damping of the NiFe layer for $t_{\mathrm{Cr}}=1,2$, and $5 \mathrm{~nm}$. For the thinnest $\mathrm{Cr}$ layer there is a strong fourfold anisotropy that correlates with the anisotropy of the CoFe, but as $t_{\mathrm{Cr}}$ increases this anisotropy is reduced, moving towards the isotropic damping expected of polycrystalline $\mathrm{NiFe}$. The fact that the anisotropy is most pronounced for thin $\mathrm{Cr}$ interlayers indicates the presence of anisotropic spin pumping. In contrast to the $\mathrm{NiFe}$, the damping in the $\mathrm{CoFe}$ layer is lower, with a corresponding reduced total anisotropy that persists for all values of $t_{\mathrm{Cr}}[31]$.

If the static magnetizations of the two FM layers are noncollinear, the efficiency of spin pumping is reduced due to the increased backflow of spin angular momentum and the partial cancellation of damping [19,20]. This can lead to an in-plane variation of spin pumping, particularly in the case of antiparallel alignment $[22,25]$. To ensure that the two magnetizations were collinear, fits to extract $\alpha$ were performed at fields above the anisotropy field of the CoFe. This was confirmed by calculations of the free energy derivative. Another possible source of anisotropic damping was outlined by Timopheev et al. [23], whereby a switch in the optical and acoustic character of the modes combined with spin pumping causes a sharp increase in damping. To exclude this effect, fits to extract $\alpha$ were restricted to the region above the crossing point of the resonances; this also avoids confusion due to antidamping arising from overlapping resonances [24]. Furthermore, the trend in anisotropy of damping as a function of $t_{\mathrm{Cr}}$ does not match the trend observed in the static coupling. The damping anisotropy follows a monotonic decrease with increasing $t_{\mathrm{Cr}}$, while $A_{\mathrm{ex}}$ changes sign and oscillates over the same range. It is important to note that the damping anisotropy is present for $t_{\mathrm{Cr}}=2 \mathrm{~nm}$, where the static exchange coupling is close to zero $\left[A_{\mathrm{ex}}=(3.3 \pm 0.6) \times 10^{-6} \mathrm{~J} \mathrm{~m}^{-2}\right]$.

These results therefore suggest that the spin pumping from the source layer can be effectively controlled by the inplane damping anisotropy of the sink layer. Transmission of the spin current at the $\mathrm{Cr} / \mathrm{CoFe}$ interface is then modified by the same mechanism that leads to anisotropic damping within the $\mathrm{CoFe}$ layer. The highest spin pumping damping occurs when the static magnetization is aligned with the direction of maximum damping in the $\mathrm{CoFe}$, along the hard axis. Damping is maximized along the hard axis, as most of the pumped spins are absorbed by the $\mathrm{CoFe}$, which acts as a spin sink for the pure spin current ejected from the onresonance NiFe layer. Along the easy axis, the spin current 

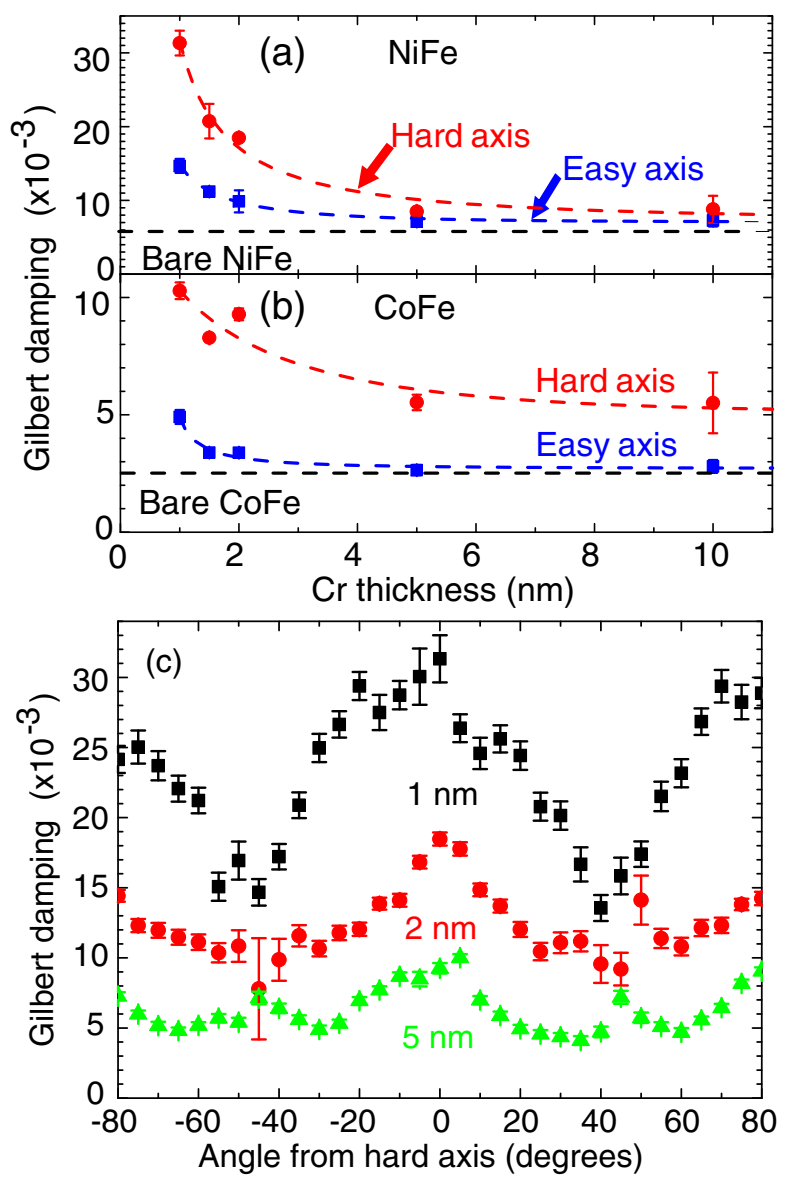

FIG. 3. Extracted total Gilbert damping for $\mathrm{NiFe}$ (a) and $\mathrm{CoFe}$ (b) layers, measured along the easy and hard axes of the $\mathrm{CoFe}$ layer. The flat line indicates the damping of the bare ferromagnets, while dashed lines are fits to the data that yield a spin diffusion length of $8 \mathrm{~nm}$. The observed anisotropy of damping is shown in more detail in (c), plotting the Gilbert damping of the $\mathrm{NiFe}$ layer as a function of bias field angle with respect to the hard axis of the $\mathrm{CoFe}$ layer.

is partially reflected at the $\mathrm{Cr} / \mathrm{CoFe}$ interface and returns to the $\mathrm{NiFe}$, where it exerts an antidamping torque on the precessing magnetization. This raises the possibility of a new mechanism for fine control of spin pumping through modification of sink layer damping parameters and engineering of the NM-FM interfaces.

Layer-resolved measurements of the magnetodynamics were performed with XFMR to separate the effects of static and dynamic exchange, and to investigate the anisotropic spin transfer in more detail. A driving frequency of $4 \mathrm{GHz}$ was used to excite precession across the $\mathrm{NiFe}$ resonance at $\sim 14 \mathrm{mT}$, while the XMCD amplitudes at the Co and Ni $L_{3}$ edges were measured. The relative phase of microwave excitation and $\mathrm{x}$-ray pulse was varied, extracting amplitude and phase of precession from plots of time-dependent magnetization $[9,27]$. The results are plotted in Fig. 4, showing the phase of precession with the bias field aligned along the easy axis [Fig. 4(a)], an intermediate axis of $22^{\circ}$ away from the easy axis [Fig. 4(b)], and along the hard axis [Fig. 4(c)]. These measurements show how the anisotropic spin pumping affects the spin transfer torque exerted on the magnetization of the CoFe layer. The expected phase shift of almost $180^{\circ}$ is observed in the on-resonance Ni data, while Co shows significant induced precession.

XFMR results can be modeled by a linearized macrospin solution of the Landau-Lifshitz-Gilbert equation, incorporating both static coupling and dynamic exchange through spin pumping $[9,24]$ :

$$
\begin{aligned}
-\frac{\partial \mathbf{m}_{i}}{\partial t}= & \gamma_{i} \mathbf{m}_{i} \times\left(\mathbf{H}_{\mathrm{eff}}^{i}+\beta_{i} M_{s, j}\right)-\left(\alpha_{i}^{0}+\alpha_{i i}^{\mathrm{sp}}\right) \frac{\partial \mathbf{m}_{i}}{\partial t} \\
& +\alpha_{i j}^{\mathrm{sp}} \mathbf{m}_{j} \times \frac{\partial \mathbf{m}_{j}}{\partial t},
\end{aligned}
$$

where the subscript denotes the magnetic layer, $\boldsymbol{\beta}_{i}$ the static exchange coupling, $\alpha_{i i}^{\mathrm{sp}}$ additional damping due to spin pumping out of layer $i$, and $\alpha_{i j}^{\mathrm{sp}}$ antidamping due to pumping into layer $i$ from layer $j$. Solving Eq. (4) yields the AC susceptibility, from which the amplitude and phase of the precession were determined. This approach uniquely determines the different contributions of static and dynamic exchange and allows increased spin pumping to be separated from increased intrinsic damping. According to this model, static coupling leads to a unipolar (absorptive) feature in the phase, while dynamic coupling leads to a bipolar (dispersive) feature, the combination of the two leads to an asymmetric steplike feature; examples are shown in Fig. 4(d). The solid lines in Figs. 4(a)-4(c) are the results from this model using parameters extracted from the analysis of Kittel curves (see Fig. 2) and angledependent damping (see Fig. 3). Close agreement with the XFMR data confirms the presence of strongly anisotropic spin pumping, as opposed to increased intrinsic damping or static exchange, and shows the importance of considering both exchange mechanisms when modeling coupled magnetodynamics. As spin pumping increases, the phase shift curvature increases, transitioning from static exchange-dominated unipolar, to dynamic exchangedominated bipolar. This behavior is indicative of an increase in STT exerted on the Co moments.

In summary, we have studied the magnetization dynamics of $\mathrm{Co}_{50} \mathrm{Fe}_{50} / \mathrm{Cr}\left(t_{\mathrm{Cr}}\right) / \mathrm{Ni}_{81} \mathrm{Fe}_{19}$ trilayers as a function of $t_{\mathrm{Cr}}$. We find a significant anisotropy of spin pumping from the NiFe layer arising from an anisotropy of Gilbert damping in the CoFe layer. This suggests that the transmission across the spacer layer is governed by the characteristics of the spin sink as well as the spin source. This anisotropy is unaffected by the strength or character of the static exchange coupling, but it is suppressed as $t_{\mathrm{Cr}}$ exceeds the spin diffusion length in $\mathrm{Cr}$, at $\sim 8 \mathrm{~nm}$. We attribute the observed angle dependence to anisotropic transmission and the reflection of spins at the $\mathrm{Cr} / \mathrm{CoFe}$ interface. Layerresolved measurements of induced precession in the $\mathrm{CoFe}$ 

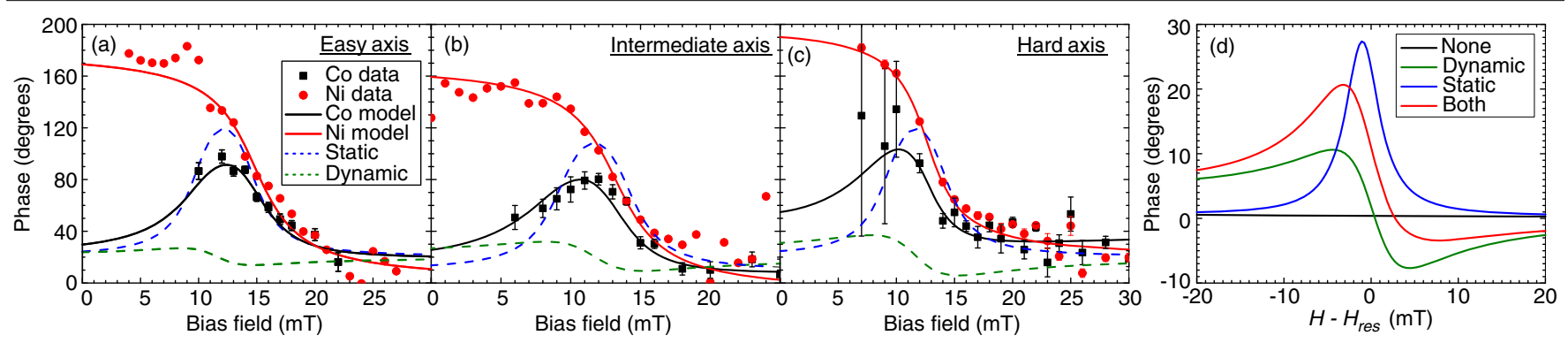

FIG. 4. Phase of precession for Co (black squares) and Ni (red circles) moments as measured by XFMR for the $t_{\mathrm{Cr}}=1 \mathrm{~nm}$ sample with the bias field along the easy (a), intermediate ( $22^{\circ}$ away) (b), and hard (c) axes of the CoFe layer. Solid lines are model calculations using parameters extracted by the fitting of Kittel curves (see Fig. 2) and the damping (see Fig. 3). Dashed lines show the effects of dynamic coupling only (green) and static coupling only (blue). The large error bars in (c) are due to a canting of magnetization reducing the projection along the beam, in turn reducing the measured XMCD signal. (d) Differing effects of static and dynamic exchange on induced precession. Note that the couplings can also interfere with each other, e.g., the increased linewidth from the dynamic interaction leads to a larger phase shift from the static interaction. The dashed lines corresponding to static only are for a spin valve with no spin pumping, and therefore they are the same for all magnetization orientations. In the case of the dynamic-only dashed lines, turning off the static coupling causes the resonance field to shift by a few milliteslas; this effect has been removed for ease of comparison.

at the $\mathrm{NiFe}$ resonance at $4 \mathrm{GHz}$ confirm this observation, revealing the competition between static and dynamic exchange that occurs in such spin valves. These results shed new light on the increasingly important topic of the anisotropic generation and detection of spin currents. Furthermore, they suggest the possibility of a further control of spin pumping through magnetization alignment, while also suggesting new concepts to manipulate spin pumping through modification of the damping mechanisms in the spin sink layer.

Beam time awarded on $\mathrm{I} 10$ at the Diamond Light Source (Proposal No. SI-11501) and 4.0.2 at the Advanced Light Source is acknowledged. A. A. B. was supported by the Diamond Light Source and EPSRC through a Doctoral Training Award. The beam line I05 team is thanked for hosting the mini-MBE instrument and for their technical assistance.

*thorsten.hesjedal@physics.ox.ac.uk † Gerrit.vanderLaan@diamond.ac.uk

[1] S. Urazhdin, V. E. Demidov, H. Ulrichs, T. Kendziorczyk, T. Kuhn, J. Leuthold, G. Wilde, and S. O. Demokritov, Nanomagnonic devices based on the spin-transfer torque, Nat. Nanotechnol. 9, 509 (2014).

[2] N. Locatelli, V. Cros, and J. Grollier, Spin-torque building blocks, Nat. Mater. 13, 11 (2014).

[3] J. C. Sankey, Y.-T. Cui, J. Z. Sun, J. C. Slonczewski, R. A. Buhrman, and D.C. Ralph, Measurement of the spintransfer-torque vector in magnetic tunnel junctions, Nat. Phys. 4, 67 (2008).

[4] A. Brataas, Y. Tserkovnyak, G. E. W. Bauer, and B. I. Halperin, Spin battery operated by ferromagnetic resonance, Phys. Rev. B 66, 060404 (2002).

[5] M. D. Stiles and A. Zangwill, Anatomy of spin-transfer torque, Phys. Rev. B 66, 014407 (2002).
[6] S. Mizukami, Y. Ando, and T. Miyazaki, Effect of spin diffusion on Gilbert damping for a very thin permalloy layer in $\mathrm{Cu} /$ permalloy/Cu/Pt films, Phys. Rev. B 66, 104413 (2002).

[7] Y. Tserkovnyak, A. Brataas, and G. E. W. Bauer, Spin pumping and magnetization dynamics in metallic multilayers, Phys. Rev. B 66, 224403 (2002).

[8] O. Mosendz, J. E. Pearson, F. Y. Fradin, S. D. Bader, and A. Hoffmann, Suppression of spin-pumping by a $\mathrm{MgO}$ tunnelbarrier, Appl. Phys. Lett. 96, 022502 (2010).

[9] M. K. Marcham, L. R. Shelford, S. A. Cavill, P. S. Keatley, W. Yu, P. Shafer, A. Neudert, J. R. Childress, J. A. Katine, E. Arenholz, N. D. Telling, G. van der Laan, and R. J. Hicken, Phase-resolved x-ray ferromagnetic resonance measurements of spin pumping in spin valve structures, Phys. Rev. B 87, 180403 (2013).

[10] A. A. Baker, A. I. Figueroa, L. J. Collins-McIntyre, G. van der Laan, and T. Hesjedal, Spin pumping in ferromagnettopological insulator-ferromagnet heterostructures, Sci. Rep. 5, 7907 (2015).

[11] R. Kukreja, S. Bonetti, Z. Chen, D. Backes, Y. Acremann, J. A. Katine, A. D. Kent, H. A. Dürr, H. Ohldag, and J. Stöhr, X-ray Detection of Transient Magnetic Moments Induced by a Spin Current in $\mathrm{Cu}$, Phys. Rev. Lett. 115, 096601 (2015).

[12] Y. Tserkovnyak, A. Brataas, G. E. W. Bauer, and B. I. Halperin, Nonlocal magnetization dynamics in ferromagnetic heterostructures, Rev. Mod. Phys. 77, 1375 (2005).

[13] J. M. Shaw, H. T. Nembach, and T. J. Silva, Determination of spin pumping as a source of linewidth in sputtered $\mathrm{Co}_{90} \mathrm{Fe}_{10} / \mathrm{Pd}$ multilayers by use of broadband ferromagnetic resonance spectroscopy, Phys. Rev. B 85, 054412 (2012).

[14] C. H. Du, H. L. Wang, Y. Pu, T. L. Meyer, P. M. Woodward, F. Y. Yang, and P. C. Hammel, Probing the Spin Pumping Mechanism: Exchange Coupling with Exponential Decay in $\mathrm{Y}_{3} \mathrm{Fe}_{5} \mathrm{O}_{12} /$ Barrier/Pt Heterostructures, Phys. Rev. Lett. 111, 247202 (2013).

[15] B. Kardasz and B. Heinrich, Ferromagnetic resonance studies of accumulation and diffusion of spin momentum 
density in $\mathrm{Fe} / \mathrm{Ag} / \mathrm{Fe} / \mathrm{GaAs}(001)$ and $\mathrm{Ag} / \mathrm{Fe} / \mathrm{GaAs}(001)$ structures, Phys. Rev. B 81, 094409 (2010).

[16] G. Woltersdorf, M. Buess, B. Heinrich, and C. H. Back, Time Resolved Magnetization Dynamics of Ultrathin $\mathrm{Fe}$ (001) Films: Spin-Pumping and Two-Magnon Scattering, Phys. Rev. Lett. 95, 037401 (2005).

[17] J. Lindner, K. Lenz, E. Kosubek, K. Baberschke, D. Spoddig, R. Meckenstock, J. Pelzl, Z. Frait, and D. L. Mills, Non-Gilbert-type damping of the magnetic relaxation in ultrathin ferromagnets: Importance of magnon-magnon scattering, Phys. Rev. B 68, 060102 (2003).

[18] Kh. Zakeri, J. Lindner, I. Barsukov, R. Meckenstock, M. Farle, U. von Hörsten, H. Wende, W. Keune, J. Rocker, S. S. Kalarickal, K. Lenz, W. Kuch, K. Baberschke, and Z. Frait, Spin dynamics in ferromagnets: Gilbert damping and twomagnon scattering, Phys. Rev. B 76, 104416 (2007).

[19] T. Taniguchi and H. Imamura, Enhancement of the Gilbert damping constant due to spin pumping in noncollinear ferromagnet/nonmagnet/ferromagnet trilayer systems, Phys. Rev. B 76, 092402 (2007).

[20] J.-V. Kim and C. Chappert, Magnetization dynamics in spinvalve structures with spin pumping, J. Magn. Magn. Mater. 286, 56 (2005).

[21] T. Chiba, G. E. W. Bauer, and S. Takahashi, Magnetization damping in noncollinear spin valves with antiferromagnetic interlayer couplings, Phys. Rev. B 92, 054407 (2015).

[22] X. Joyeux, T. Devolder, J.-V. Kim, Y. G. de la Torre, S. Eimer, and C. Chappert, Configuration and temperature dependence of magnetic damping in spin valves, J. Appl. Phys. 110, 063915 (2011).

[23] A. A. Timopheev, Yu. G. Pogorelov, S. Cardoso, P. P. Freitas, G. N. Kakazei, and N.A. Sobolev, Dynamic exchange via spin currents in acoustic and optical modes of ferromagnetic resonance in spin-valve structures, Phys. Rev. B 89, 144410 (2014).

[24] B. Heinrich, Y. Tserkovnyak, G. Woltersdorf, A. Brataas, R. Urban, and G. E. W. Bauer, Dynamic Exchange Coupling in Magnetic Bilayers, Phys. Rev. Lett. 90, 187601 (2003).

[25] R. Salikhov, R. Abrudan, F. Brüssing, K. Gross, C. Luo, K. Westerholt, H. Zabel, F. Radu, and I. A. Garifullin, Configurational dependence of the magnetization dynamics in spin valve systems: Influence of spin pumping and domain wall induced coupling, Phys. Rev. B 86, 144422 (2012).

[26] A. A. Baker, W. Braun, G. Gassler, S. Rembold, A. Fischer, and T. Hesjedal, An ultra-compact, high-throughput molecular beam epitaxy growth system, Rev. Sci. Instrum. 86, 043901 (2015).

[27] A. I. Figueroa, A. A. Baker, L. J. Collins-McIntyre, T. Hesjedal, and G. van der Laan, Spin pumping through a topological insulator probed by x-ray detected ferromagnetic resonance, J. Magn. Magn. Mater. 400, 178 (2016).

[28] C. Kittel, On the theory of ferromagnetic resonance absorption, Phys. Rev. 73, 155 (1948).

[29] D. A. Arena, E. Vescovo, C.-C. Kao, Y. Guan, and W. E. Bailey, Weakly coupled motion of individual layers in ferromagnetic resonance, Phys. Rev. B 74, 064409 (2006).

[30] G. B. G. Stenning, L. R. Shelford, S. A. Cavill, F. Hoffmann, M. Haertinger, T. Hesjedal, G. Woltersdorf, G. J. Bowden, S. A. Gregory, C. H. Back, P. A. J. de Groot, and G. van der Laan, Magnetization dynamics in an exchange-coupled $\mathrm{NiFe} / \mathrm{CoFe}$ bilayer studied by $\mathrm{x}$-ray detected ferromagnetic resonance, New J. Phys. 17, 013019 (2015).

[31] See Supplemental Material at http://link.aps.org/ supplemental/10.1103/PhysRevLett.116.047201 for further information on magnetocrystalline anisotropy parameters, linewidth data fitting, and angle-dependent damping in CoFe layer.

[32] R. Topkaya, M. Erkovan, A. Öztürk, O. Öztürk, B. Aktaş, and M. Özdemir, Ferromagnetic resonance studies of exchange coupled ultrathin $\mathrm{Py} / \mathrm{Cr} / \mathrm{Py}$ trilayers, J. Appl. Phys. 108, 023910 (2010).

[33] S. S. Kalarickal, P. Krivosik, M. Wu, C. E. Patton, M. L. Schneider, P. Kabos, T. J. Silva, and J. P. Nibarger, Ferromagnetic resonance linewidth in metallic thin films: Comparison of measurement methods, J. Appl. Phys. 99, 093909 (2006).

[34] C. Du, H. Wang, F. Yang, and P. C. Hammel, Systematic variation of spin-orbit coupling with $d$-orbital filling: Large inverse spin Hall effect in $3 d$ transition metals, Phys. Rev. B 90, 140407 (2014).

[35] G. Woltersdorf and B. Heinrich, Two-magnon scattering in a self-assembled nanoscale network of misfit dislocations, Phys. Rev. B 69, 184417 (2004). 